\title{
Direct evidence of atomic-scale structural fluctuations in catalyst nanoparticles
}

$3 \operatorname{tarajan}^{1,2,4}$ and Renu Sharma ${ }^{1}$.

$4 \quad{ }^{1}$ Center for Nanoscale Science and Technology, National Institute of Standards and Technology, Gaithersburg, MD 5 20899-6203, USA

$6 \quad{ }^{2}$ University of Maryland - IREAP, College Park, MD 20742, USA

$7 \quad{ }^{3}$ Department of Chemical Engineering, Texas A\&M University, College Station, TX 77843 USA

$8{ }^{4}$ Materials Measurement Laboratory, National Institute of Standards and Technology, Gaithersburg, MD 2089996203 , USA

11 *Corresponding Author: renu.sharma@nist.gov

$\dagger$ Current address: National Applied Research Lab, 14F, No. 106, Sec. 2, Heping Rd., Taipei, 10636 Taiwan (R.O.C)

KEYWORDS: Catalyst structural fluctuations, Atomic scale observations, Environmental nisms. 
ABSTRACT: Rational catalyst design requires an atomic scale mechanistic understanding of

2 the chemical pathways involved in the catalytic process. A heterogeneous catalyst typically works by

3 adsorbing reactants onto its surface, where the energies for specific bonds to dissociate and/or combine

4 with other species (to form desired intermediate or final products) are lower. Here, using the catalytic

5 growth of single-walled carbon nanotubes (SWCNTs) as a prototype reaction, we show that the chemi-

6 cal pathway may in-fact involve the entire catalyst particle, and can proceed via the fluctuations in the

7 formation and decomposition of metastable phases in the particle interior. We record in situ and at atom-

8 ic resolution, the dynamic phase transformations occurring in a Cobalt catalyst nanoparticle during

9 SWCNT growth, using a state-of-the-art environmental transmission electron microscope (ETEM). The

10 fluctuations in catalyst carbon content are quantified by the automated, atomic-scale structural analysis

11 of the time-resolved ETEM images and correlated with the SWCNT growth rate. We find the fluctua-

12 tions in the carbon concentration in the catalyst nanoparticle and the fluctuations in nanotube growth

13 rates to be of complementary character. These findings are successfully explained by reactive molecular

14 dynamics (RMD) simulations that track the spatial and temporal evolution of the distribution of carbon

15 atoms within and on the surface of the catalyst particle. We anticipate that our approach combining real-

16 time, atomic-resolution image analysis and molecular dynamics simulations will facilitate catalyst de-

17 sign, improving reaction efficiencies and selectivity towards the growth of desired structure 


\section{INTRODUCTION}

Revealing the mechanisms by which nanometer sized catalysts act in chemical reactions and material syntheses is essential for the precise control of reaction rate, product selectivity, and ultimately, catalyst design. For example, based on the existing understanding of heterogeneous catalysis, it is assumed that the role of the catalyst in the synthesis of carbon nanotubes (CNTs) by catalytic chemical vapor deposition (C-CVD), is to decompose adsorbed carbon precursors and act as a template for CNT (graphene) nucleation. $[1,2]$ However, the detailed steps leading to graphene nucleation and subsequent nanotube growth have not been experimentally established. An alternative hypothesis, suggested by Baker et al. [3], and later supported by molecular dynamics simulations[4], is that the catalyst acts as a reservoir, dissolving carbon atoms in a liquid phase that precipitates $\mathrm{C}$ in a solid form (carbon fibers) after reaching a supersaturated concentration, analogous to the vapor-liquid-solid (VLS) mechanism proposed for silicon nanowire growth [5]. However, in situ high-resolution transmission electron microscopy (HRTEM) studies have shown that the catalyst particles are crystalline, and not liquid, during CNT growth [6-9]. These results suggest a vapor-solid-solid (VSS) mechanism, where the carbon atoms

may diffuse on the solid catalyst surface, to form the nanotube without dissolving into the catalyst $[6$, 10]. However, if dissolution of $\mathrm{C}$ into the catalyst particle were to occur, there are two possibilities: 1] formation of a stable carbide, leading to inactivation of the catalyst [11], or 2] formation of a solid solution or metastable carbides that allow for continued catalytic activity [12-15]. In this latter scenario, the carbon in the catalyst nanoparticle represents a reactive intermediate that can combine with surface carbon.

Recently, metal catalyst particles have been reported to convert to a carbide phase prior to CNT growth $[15],[13,16]$. Several theoretical studies on nucleation and growth of CNT using various simulation techniques [17] including reactive molecular dynamics [18-20], tight binding [21, 22], and timestamped force-biased Monte Carlo simulations [23] of Ni and Co catalysts have suggested that carbon 
1 atoms diffusing in and out of metal particles are present at different stages of the process, thereby pro-

2 viding evidence that carbon atoms indeed diffuse into the catalyst bulk prior to incorporating into

3 SWCNTs.. Structural heterogeneity for cobalt catalyst nanoparticles during triple wall carbon nanotube

4 growth has also been reported [14]. The lower energy of M-C bond formation, for example, $2.6 \mathrm{eV}$ for

5 Ni-C compared to $6.6 \mathrm{eV}$ for $\mathrm{C}-\mathrm{C}$ bonds $[24,25]$, and the stronger cohesion energy per formula unit of

6 Ni-C (10.1 eV) compared to Ni-Ni (4.5 eV) and C-C (7.4 e) [22] favors metal carbide formation over

7 graphene as explained in an earlier report [18]. However, the existence of carbide structures raises fur-

8 ther questions concerning the role of the catalyst in nucleating CNTs: does carbon diffuse on the surface

9 of the metal carbide or via the bulk, as proposed for metal particles? Or do metastable carbide struc-

10 tures, formed under non-equilibrium conditions, play a part?

11 By employing an in situ environmental transmission electron microscope (ETEM) as an experi-

12 mental platform and reactive molecular dynamics (RMD) simulations [26],[27], we resolve the entire

13 process from the nucleation and growth of a SWCNT from a single catalyst nanoparticle to termination

14 (catalyst deactivation). Here, we present the observed and simulated time-resolved structural dynamics

15 of catalyst nanoparticles under non-equilibrium conditions during SWCNT growth and compare the

16 chemical changes associated with phase fluctuations to the carbon incorporation rate in growing nano-

17 tubes. Intriguingly, we identify two sources of carbon supporting the SWCNT growth 1] Surface diffus-

18 ing $\mathrm{C}$ atoms that are incorporated at a constant rate 2] $\mathrm{C}$ atoms diffusing out of metastable carbides in

19 the particle bulk, incorporated at fluctuating time intervals. Our results hold relevance for a number of

20 other chemical reactions where Co nanoparticles are used as active catalysts for e.g. Ficsher Tropsch

21 process for hydrocarbon synthesis and petroleum cracking [28]. A broader implication of this study is

22 that our methods may be employed to resolve dynamic atomic-level processes in heterogeneous cataly-

23 sis. 

13 earlier [15].

4

\section{EXPERIMENTAL SECTION}

\subsection{ETEM experiments.}

Atomic-resolution videos of SWCNT growth were recorded using an environmental transmission electron microscope (ETEM), operated at $300 \mathrm{kV}$ and equipped with an image corrector, at a rate of 10 frames per s. Acetylene $\left(\mathrm{C}_{2} \mathrm{H}_{2}\right)$ and $\mathrm{Co}_{\mathrm{x}} \mathrm{Mo}_{1-\mathrm{x}} \mathrm{MgO}$ (prepared by wet chemical methods) [29] were used as carbon precursors and a catalyst-support system, respectively. The catalyst-support system was first heated to $750{ }^{\circ} \mathrm{C}$ in oxygen $(100 \mathrm{~Pa})$ to remove impurities such as gaseous hydrocarbons adsorbed on the surface. Then the sample was cooled to the SWCNT growth temperature of $650{ }^{\circ} \mathrm{C}$ in vacuum (0.0001 Pa), and was maintained at this temperature for 10 minutes before exposing to $0.01 \mathrm{~Pa}$ of $\mathrm{C}_{2} \mathrm{H}_{2}$. Crystalline Co clusters precipitated from the support and formed $2 \mathrm{~nm}$ to $5 \mathrm{~nm}$ sized particles. These particles nucleated and grew SWCNTs. No molybdenum was found within the nanoparticles as reported

\subsection{Simulation Methods.}

Classical reactive molecular dynamics (RMD) simulations as implemented in our SIMCAT code [25] were employed to simulate the catalyst particle during SWCNT growth. was employed to simulate the catalyst particle during SWCNT growth. The model consists of a carbide-like particle $(1.8 \mathrm{~nm}$ diameter, 160 atoms) with $\mathrm{Co}_{2} \mathrm{C}$ composition deposited on a rectangular monolayer substrate model in an atmosphere of a $\mathrm{C}$ precursor gas model $(\mathrm{P} \approx 18,000 \mathrm{~Pa}$, corresponding to $1 \mathrm{C}$ atom in the box $)$. The simulation box was $3.98 \mathrm{~nm}$ long, $3.88 \mathrm{~nm}$ wide, and $50 \mathrm{~nm}$ deep. The reactive empirical bond order (REBO) Brenner potential [30], which is based on the Tersoff [31] and Tersoff-Brenner potentials [32], was used to represent carbon-carbon interactions. Metal-carbon interactions using [25, 31] a DFTparameterized potential based on a many-body scheme based on the Tersoff potential that combines 
1 Morse-type repulsive and attractive terms with an additional screening parameter; which takes into ac-

2 count the coordination of $\mathrm{C}$ atoms to distinguish between surface atoms and dissolved $\mathrm{C}$ atoms, and dif-

3 ferent hybridization states. The many-body Sutton-Chen potential was used to describe the metal-metal

4 interaction [33] and the metal-substrate interaction was parameterized using an average adsorption

5 strength of $6.11 \mathrm{eV} \cdot \mathrm{nm}^{-2}$. A predictor-corrector algorithm and a time step of $0.5 \mathrm{fs}$ were used in the inte-

6 gration of the equations of motion and the temperature was maintained at $650{ }^{\circ} \mathrm{C}$ using the Nosé-

7 Hoover thermostat [25]. The dissolution residence time $\left(\tau_{\mathrm{D}}\right)$ is the time that a $\mathrm{C}$ atom remains dissolved

8 in the particle. A number of metal first nearest-neighbor (MNN) greater than or equal to $5(\mathrm{MNN} \geq 5)$

9 was used as criterion to determine whether $\mathrm{C}$ atoms were dissolved or not. $\mathrm{C}$ atoms that do not dissolve 10 in the particle $\left(\tau_{\mathrm{D}}=0 \mathrm{~ns}\right)$ are labeled as $\mathrm{SD}$, those remaining dissolved for less than $3 \mathrm{~ns}\left(0.001 \mathrm{~ns} \leq \tau_{\mathrm{D}}\right.$

$11 \leq 3 \mathrm{~ns})$ as $\mathrm{BD}$, and those remaining dissolved for longer ( $\left.\tau_{\mathrm{D}} \geq 3 \mathrm{~ns}\right)$ as $\mathrm{CF}$.

\section{RESULTS AND DISCUSSION:}

\subsection{Environmental TEM:}

16 Figure 1a is a frame extracted from a video (Movie S1, frame rate of $10 \mathrm{~s}^{-1}$ ) of SWCNT growth

17 from a $(2.5 \pm 0.04) \mathrm{nm}$ diameter Co catalyst particle (uncertainties represent the deviation from a per-

18 fectly spherical shape) supported on $\mathrm{MgO}$. As reported earlier, the metal particle converts to a carbide

19 phase with continued exposure to $\mathrm{C}_{2} \mathrm{H}_{2}$, before nucleating a SWCNT [15] The video sequence used here

20 was captured after the nucleation of a SWCNT with its rim anchored to the particle, enclosing approx-

21 imately one-third of the top portion of the particle (marked by arrows in Figure 1a and 1d). Within this

22 individual $(2.5 \pm 0.04) \mathrm{nm}$ diameter particle, two distinct regions with different structures and a clear

23 boundary, can be visually identified (Figure 1a and 1d): a region of the particle that is mostly enclosed

24 in the SWCNT (R1), and the rest of the particle (R2). By measuring lattice spacings and angles from the 
1 fast Fourier transforms (FFTs) of the high-resolution images (Figure 1b and 1c), the structures of the

2 regions $\mathrm{R} 1$ and $\mathrm{R} 2$ are identified as $\mathrm{Co}$ metal and $\mathrm{Co}_{2} \mathrm{C}$, respectively (Figure S5 and Table S1). While

3 the co-existence of $\mathrm{Co}, \mathrm{Co}_{2} \mathrm{C}$ and other related phases in an active catalyst particle has been reported

4 [14], 'direct evidence' in the images showing two structures with a pronounced boundary has not been

5 reported before. Ex situ electron diffraction studies of Co have also reported the formation of $\mathrm{Co}_{3} \mathrm{C}$ and

$6 \mathrm{Co}_{2} \mathrm{C}$ at $450{ }^{\circ} \mathrm{C}$ and $500{ }^{\circ} \mathrm{C}$, respectively, that decomposed to Co upon heating above $650{ }^{\circ} \mathrm{C}$ [34]. It is

7 possible that such metastable structures with clear boundaries co-exist in nanoparticles under non-

8 equilibrium conditions and can only be revealed by high resolution images acquired under reaction con-

9 ditions.

10 In addition to the co-existence of these two distinct structures, their respective areas (R1 and R2)

11 are observed to fluctuate during SWCNT growth (Figure 1e-h). In order to quantitatively evaluate the

12 relative areas of R1 and R2, and their evolution with time, careful measurements of distances between

13 individual atomic columns in each frame are required. We establish an image processing scheme (IPS)

14 that locates the atomic-column positions in each frame, measures the average first nearest-neighbor dis-

15 tance for every individual column, and then assigns atomic columns as belonging to the Co metal $(0.22$

$16 \mathrm{~nm}$ ) or Co-carbide $(0.25 \mathrm{~nm})$ phases accordingly [35] (Figure S6). The high precision of the IPS (7 pm

17 and $15 \mathrm{pm}$ for Co and Co-carbide respectively) enables the accurate assignment of phases and identifi-

18 cation of phase boundaries [35]

19 This quantification finds the size of the Co area (R1) at $4.0 \mathrm{~s}$ to be approximately 1.33 times

20 larger (Figure 1e) than that at $10.0 \mathrm{~s}$ (Figure 1f). After $10.0 \mathrm{~s}$, the size of this area increases again (Fig-

21 ure 1g), and then decreases at $40.0 \mathrm{~s}$ (Figure 1h). The variation in sizes of R1 can be attributed to the

22 difference between the rate of carbide decomposition ( $\mathrm{r}_{1}$, equation 1) and formation $\left(\mathrm{r}_{2}\right.$, equation 2$)$.

23 This leads to a decrease $(x \mathrm{C})$ or increase $(y \mathrm{C})$ in the total carbon amount $(\Delta(t))$ in the particle (equation

24 3) under non-equilibrium conditions. 
1

2

3

4

5 given time. We first carefully measure the carbon distribution within the particle in one frame (Figure

6 2a). The ratios of carbon to $\mathrm{Co}(\mathrm{C}: \mathrm{Co})$ in each atomic layer are shown in Figure $2 \mathrm{~b}$, where layer 1 is in

7 contact with the $\mathrm{MgO}$ support and layer 10 is the top surface that is enclosed by the growing SWCNT

8 (red arrow). We find that the C:Co ratio is 0.5 in the first three atomic layers, and then gradually drops

9 down to 0.31 in the next five layers. For the $9^{\text {th }}$ and $10^{\text {th }}$ atomic layers, the ratios for are approximately

$10 \quad 0.16$ (Figure 2b).
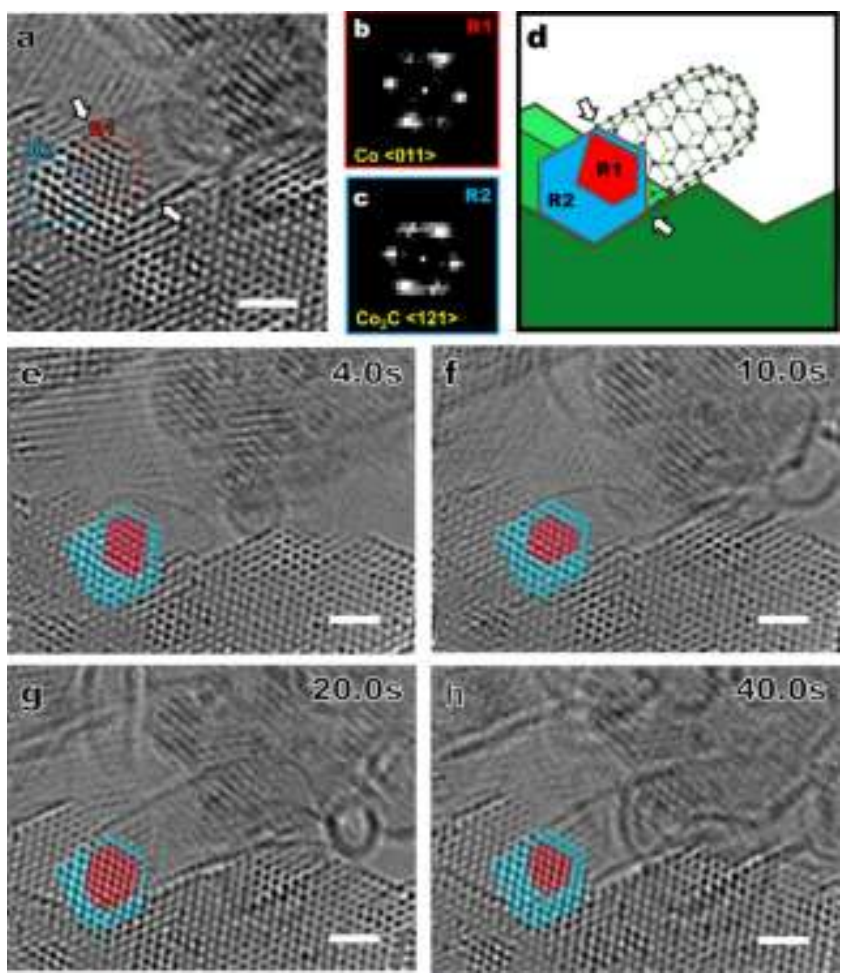

13 Figure 1. Co and $\mathrm{Co}_{2} \mathrm{C}$ domains in catalyst nanoparticle and dynamic fluctuations in their relative areas.

14 (a) A high-resolution image of a catalyst particle during SWCNT growth that contains two distinct re- 
1 gions, R1 and R2. (b) and (c) FFT from R1 and R2 showing the structures in R1 and R2 as Co and $2 \quad \mathrm{Co}_{2} \mathrm{C}$, respectively. (d) A simplified illustration of Figure 1a identifying the catalyst particle with the 3 two structures R1 and R2, in contact along one edge with the $\mathrm{MgO}$ substrate, which is in the same plane 4 (dark green). $\mathrm{MgO}$ planes below the particle, are marked as lighter shades of green. (e-h) High5 resolution snapshots of CNT growth at $4.0 \mathrm{~s} \mathrm{(e),} 10.0 \mathrm{~s}$ (f), $20.0 \mathrm{~s}(\mathrm{~g})$, and $40.0 \mathrm{~s}$ (h). Co (R1) and Co6 carbide (R2) areas are colored red and blue, respectively, to highlight the change in respective areas with 7 time. All scale bars represent $1 \mathrm{~nm}$.

Experimentally, the number of carbon atoms in the nanoparticle at time $t\left(\mathrm{C}_{\mathrm{p}}(\mathrm{t})\right)$ can be obtained

10 from individual frames of the growth video by measuring the volume of the $\mathrm{Co}_{2} \mathrm{C}$ region, relative to the

11 volume of the catalyst particle (Figure $\mathrm{S6}$ ). Figure $2 \mathrm{c}$ shows the variation in $\mathrm{C}_{\mathrm{p}}(\mathrm{t})$ as a function of time.

12 The amount of carbon inside the particle fluctuates aperiodically until the supply of carbon precursor is

13 ended. For instance, the amount of carbon drops to $(163 \pm 3)$ atoms at $3.6 \mathrm{~s}$, increases again to $(210 \pm 3)$

14 atoms at $9.8 \mathrm{~s}$, and then drops to $(162 \pm 3)$ atoms at $16.5 \mathrm{~s}$. The uncertainty in $\mathrm{C}_{\mathrm{p}}(\mathrm{t})$ is derived by propa-

15 gating the uncertainty in atomic position identification (15 pm) through equations shown in the Supple-

16 mentary Information. In order to elucidate the role of carbide phase for the SWCNT growth, we meas-

17 ure relative change in the length of the growing SWCNT with time. Since the diameter of the growing

18 SWCNT remains relatively unchanged during this observation period, we obtain number of the carbon

19 atoms added during a specific time period by measuring the increase in the arc length of the tube profile

20 (Figure S7) in each frame. The plot in Figure $2 \mathrm{~d}$ shows the variation in the number of carbon atoms

21 added to the SWCNT as a function of time. A stochastic fluctuation in the growth periods and intervals

22 between growth periods is observed and is in agreement with earlier reports.[36, 37] 

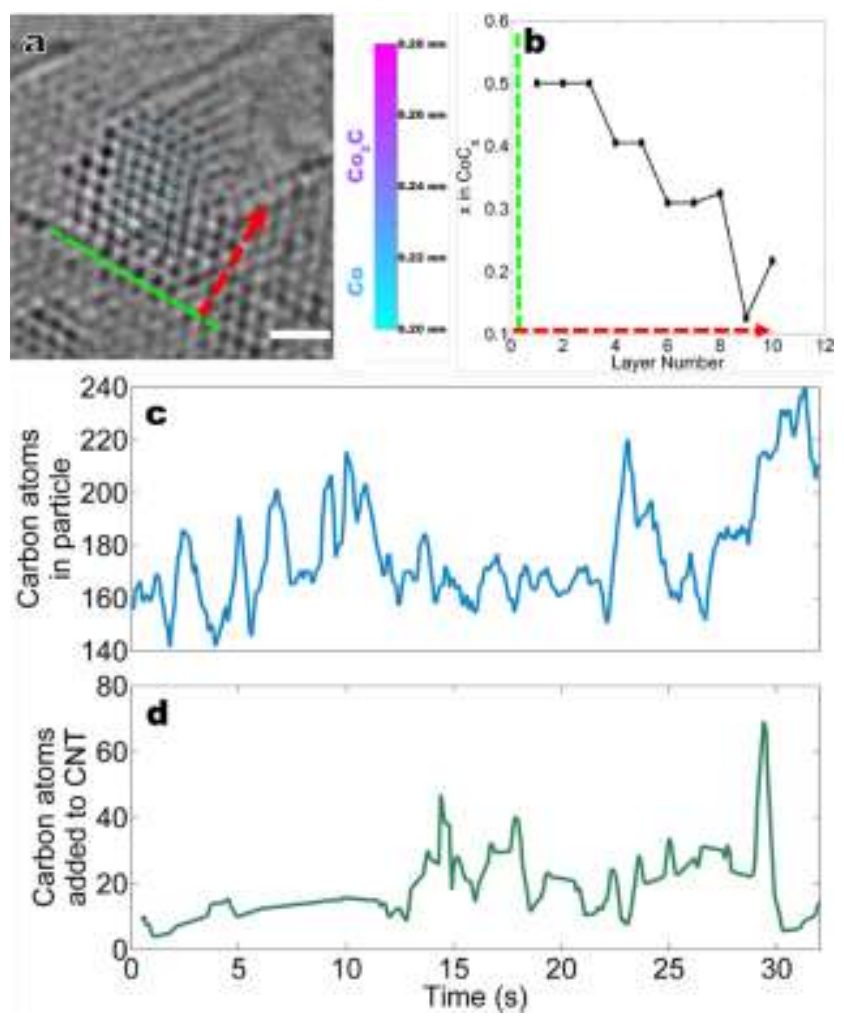

Figure 2. Experimentally measured, spatial (layer-wise) and temporal variations in carbon content in the nanoparticle and the growing SWCNT. (a) A single high resolution frame after applying IPS showing the location of atomic columns, where inter-atomic column distances ranging $0.20 \mathrm{~nm}$ to $0.22 \mathrm{~nm}$ or $0.23 \mathrm{~nm}$ to $0.26 \mathrm{~nm}$ correspond to Co or $\mathrm{Co}_{2} \mathrm{C}$, respectively. ${ }^{\text {st }}$ atomic layer of the catalyst particle is in contact with $\mathrm{MgO}$ support (green dashed line), and the $10^{\text {th }}$ layer is inside the SWCNT. Atomic layers are numbered 1 to 10 away from the green line. The scale bar represents $1 \mathrm{~nm}$. (b) Corresponding local ratios of carbon and Co as a function of atomic layer. (c) Number of carbon atoms in the catalyst particle $\mathrm{C}_{\mathrm{p}}(\mathrm{t})$; and $(\mathrm{d})$ the number of carbon atoms added to growing nanotube plotted as a function of time. A moving average of 10 frames is applied to smooth the data (raw data in Figure S8).

The carbon amount in the catalyst particle and in the tube at specific time intervals can be compared from the data provided in Figure $2 \mathrm{c}$ and $2 \mathrm{~d}$. As the number of carbon atoms added to the tube increases in the time period between $10.0 \mathrm{~s}$ to $15 \mathrm{~s}$ (Figure $2 \mathrm{~d}), \mathrm{C}_{\mathrm{p}}(\mathrm{t})$ decreases (Figure $2 \mathrm{c}$ ). On the other

14 hand, $\mathrm{C}_{\mathrm{p}}(\mathrm{t})$ increases until $23.8 \mathrm{~s}$ while the tube growth rate decreases to a local minimum. These com-

15 parisons suggest that the variations in the particle composition, i.e. low and high carbon concentration,

16 in the measured time period might be inversely related to SWCNT growth rate (Figure 2c-d). The com-

17 plementary character of the fluctuations in SWCNT growth rate and particle carbon content implies that 
1 fluctuations in the SWCNT growth could be related to the periods of carbide formation and carbide de-

2 composition.

3

4

5

6 we examine the trajectories of individual $\mathrm{C}$ atoms in a Co nanoparticle by reactive molecular dynamics

\subsection{Reactive Molecular Dynamics.}

In order to better relate the carbide formation and decomposition reactions to the tube growth, simulations (RMD) and identify the role of different types of $\mathrm{C}$ atoms and their contribution to the growth process. The RMD simulations were performed on a model $1.8 \mathrm{~nm}$ diameter $\mathrm{Co}_{2} \mathrm{C}$ particle deposited on a rectangular monolayer substrate in an atmosphere of a $\mathrm{C}$ precursor gas model $(\mathrm{P} \approx 18,000 \mathrm{~Pa}$, Figure 3a). The particle size was intentionally kept smaller and the reaction conditions, such as carbon precursor pressure, more aggressive in the simulations than in the experiment in order to decrease the calculation time needed to observe complete events from the simulations. We note that recent advances on accelerated molecular dynamics and hyperdynamics simulations have been reported and new methodologies are currently being developed such as collective variable-driven hyperdynamics (CVHD) [38] or hybrid approaches that combine reactive molecular dynamics with time-stamp force-bias Monte Carlo simulations [39], both of which could satisfactorily reproduce the dynamic behavior of reactive systems up to time scales of seconds without altering reaction conditions [38]. Previous studies using the current approach have shown that the mechanisms of nucleation and growth are the same for different particle sizes [18]. The kinetics are also analogous for different particle sizes [40] and the carbon precursor concentration [41]. Thus, we can qualitatively compare our simulations to the experiment results. Using a dissolution residence-time criterion, as reported previously [40], C atoms can be classified into three types: 1] atoms that are predominantly involved in diffusion on the particle surface (SD), or 2] in the bulk (BD) before becoming part of the growing nanotube structure or 3] atoms that remain dissolved 
1 in the nanoparticle for prolonged periods of time, which makes them candidates for participation in car2 bide formation (CF) (Figure $\mathrm{S} 1$ ).

After the nanotube cap is formed and the nucleation stage concluded, the carbon concentration in each atomic layer is obtained by calculating the ratio of z-density profiles for each atomic species (Co 5 and C) in each layer (Figure S2). The C:Co ratios from each atomic layer are averaged over 5 ns in three 6 different reaction time zones ( $35 \mathrm{~ns}$ to $40 \mathrm{~ns}, 40 \mathrm{~ns}$ to $45 \mathrm{~ns}$ and $45 \mathrm{~ns}$ to $50 \mathrm{~ns}$ ). In each time zone, the 7 ratio decreases linearly along the normal away from the substrate plane (direction guided by the arrow 8 in Figure $3 a$ ) from 0.8 to 0.2 , and stabilizes in the $4^{\text {th }}$ layer at approximately 0.2 (Figure $3 b$ ). It is noted 9 that the carbon concentration profile corresponding to the growth stage (Figure $3 b$ ) has similarity with 10 that of the nucleation stage reported in our earlier work [27], which indicates that such carbon gradient 11 starts to be generated at earlier times and consolidates at the growth stage. This analysis does not in12 clude the pure metal layer in contact with the support, as $\mathrm{C}$ atoms undergo repulsion from the support 13 and the first peak of $\mathrm{C}$ atoms from the z-density profiles is located closer to the second Co peak than to 14 the first one (Fig. S2). Although the particle size is smaller in the simulation than in the experiments, 15 both of them show that carbon stays inside the particle, and the C:Co ratio drops moving perpendicular16 ly away from the substrate plane and remains at $\approx 0.2$ in the particle region located inside the growing 17 SWCNT (Figure $2 \mathrm{~b}$ and $3 \mathrm{~b}$ ). For the layer that is in contact with the substrate, the C:Co ratio can be as 18 high as 0.75 in the simulation, which includes $\mathrm{SD}, \mathrm{BD}$ and $\mathrm{CF}$ types of $\mathrm{C}$ atoms. This ratio is restricted 19 to a maximum value of 0.5 in the experimental measurements, as from the images we can only identify 20 the carbon amount associated with the $\mathrm{Co}_{2} \mathrm{C}$ phase and not the dissolved $\mathrm{C}$ atoms, if present. Moreover, 21 the C:Co ratio for each atomic layer in time zone $45 \mathrm{~ns}$ to $50 \mathrm{~ns}$ is generally lower than those for the 22 other two time zones; especially the ratio in layer $4(\approx 0.1$ in 45 ns to $50 \mathrm{~ns}$ time zone $)$ implying that the 23 total carbon amount in the particle varies during SWCNT growth, which agrees with our experimental 24 results. 

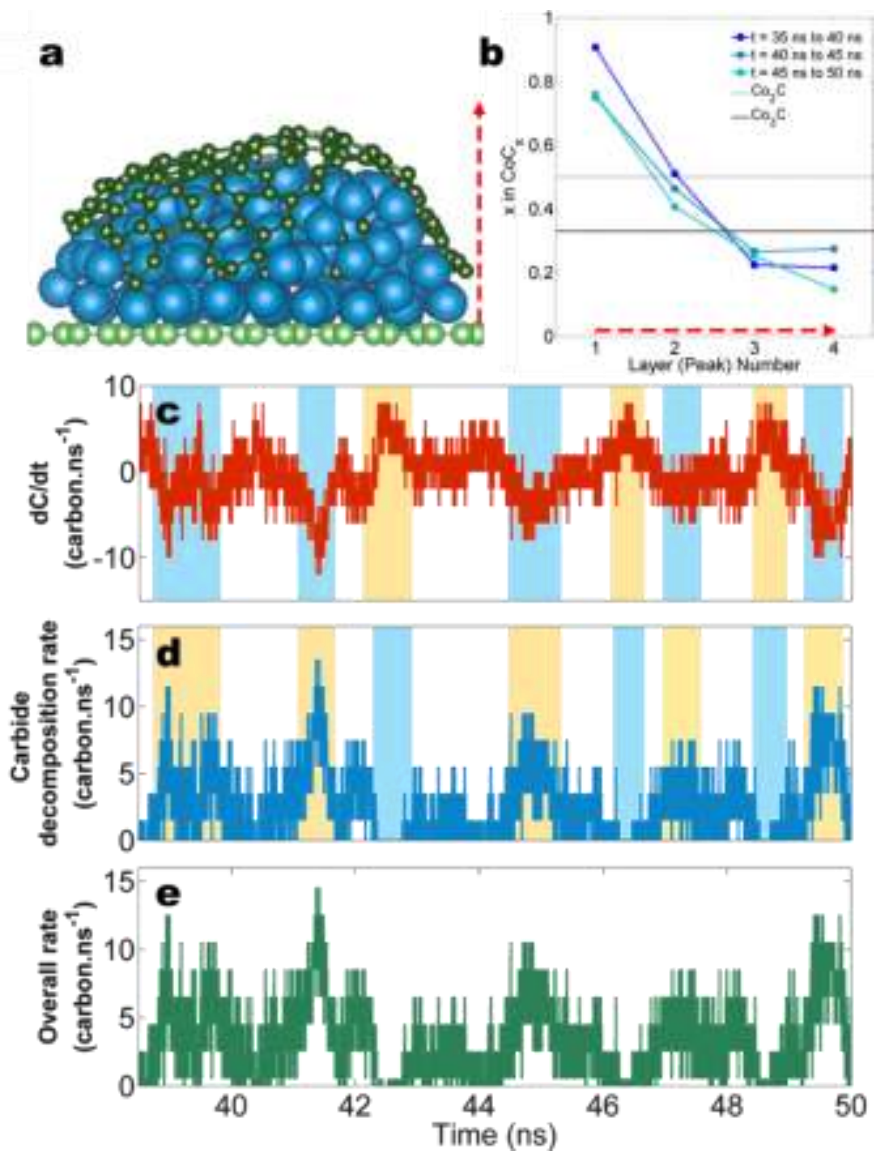

Figure 3. Simulated spatial (layer-wise) and temporal variations in carbon content in the nanoparticle and the growing SWCNT. (a) Simulated catalyst particle (on a support) that nucleates a SWCNT with atomic layers from 1 to 4 numbered in the direction of the red arrow. (b) Corresponding ratios of carbon and metal as a function of each atomic layer at different time intervals ( $35 \mathrm{~ns}$ to $40 \mathrm{~ns}$, $40 \mathrm{~ns}$ to $45 \mathrm{~ns}, 45 \mathrm{~ns}$ to $50 \mathrm{~ns}$ ). The values are obtained from z-density profiles reported in supplementary information (Figure S2). (c) Variation in the number of carbon atoms in the particle with time. (d) Carbide decomposition rate, $\mathrm{r}_{1}$, estimated from an atomic balance of carbon atoms in the particle (equation 4) versus time. Yellow or light blue regions indicate an increase or decrease, respectively, in either growth rate or carbon concentration in the particle. (e) Nanotube growth rate (surface diffusion contribution not included) estimated directly from the total number of atoms that leave the particle versus time.

To further examine the role of carbide in nanotube growth, a thorough atomic balance of $\mathrm{C}$ atoms from the RMD trajectories is performed. The $\mathrm{C}$ atomic balance equation (equation 4) shows that the carbide decomposition rate, $r_{1}$, is a function of three components. They are the carbon dissolution 
1 (carbide formation) rate, $\mathrm{r}_{2}$, (Figure S3), the variation of $\mathrm{C}$ content in the particle with time, $\mathrm{dC} / \mathrm{dt}$, and

$2 \mathrm{C}$ diffusion across the $\mathrm{C}$ gradient in the particle, $D_{c}\left(\nabla^{2} C\right)$ (Figure $\mathrm{S} 3$ ).

3

$$
r_{1}=r_{2}+\frac{d C}{d t}-D_{C} \cdot\left(\nabla^{2} C\right)
$$

As estimated from the RMD trajectories, the carbon atoms rate $\left(r_{2}\right)$ is $1.50 \cdot \mathrm{ns}^{-1}$ (Figure S4a, we note that rates reported from RMD calculations are much larger than actual experimental rates due to the accelerated nature of these simulations). In contrast, $\mathrm{dC} / \mathrm{dt}$ exhibits a fluctuating behavior in agreement with experimental observations (Figure $3 \mathrm{c}$ and $2 \mathrm{c}$, respectively). Molecular transport of $\mathrm{C}$ atoms inside the particle is described in equation 4 as the product of the diffusion coefficient of atomic carbon in the particle multiplied by the variation of the carbon concentration gradient across the nanoparticle. The diffusion term $\left(D_{c}\right)$ is estimated from the mean-squared displacement and Einstein's diffusivity equation (Figure S4b). The variation of the carbon gradient in the direction perpendicular to the substrate is estimated from the $\mathrm{C}$ composition profiles by fitting the curves in Figure $3 \mathrm{~b}$ to a quadratic function and analytically calculating the second derivative with respect to position in the z-direction. We find that the diffusion coefficient is $(1.29 \pm 0.07) \times 10^{-11} \mathrm{~m}^{2} \cdot \mathrm{s}^{-1}$, which is in agreement with reported diffusion coefficients of $\mathrm{C}$ dissolved in transition metals such as $\mathrm{Ni}$ (ranging between $10^{-11} \mathrm{~m}^{2} \cdot \mathrm{s}^{-1}$ and $10^{-10} \mathrm{~m}^{2} \cdot \mathrm{s}^{-1}$ ) [18], and the second derivative of the carbon concentration per $\mathrm{nm}^{2}$ varies between 0.14 carbon atoms and $0.23 \mathrm{C}$ atoms. Thus, by knowing $\mathrm{r}_{2}, \mathrm{dC} / \mathrm{dt}$ and $D_{c}\left(\nabla^{2} C\right), \mathrm{r}_{1}$ is obtained via equation 4 as a function of time (Figure 3d). In addition to obtaining the rates of carbide decomposition $\left(\mathrm{r}_{1}\right)$, the overall tube growth rate is also determined by monitoring independently the carbon atoms that leave the particle (Figure 3e). Despite the accelerated dynamics intrinsic to our simulation model, the results obtained are in qualitative agreement with experimental measurements. The calculated $r_{1}$ has the same fluctuations as the tube growth and its value is consistently lower than that of the tube growth by approximately 2 carbon atoms per ns. This observation is in qualitative agreement with experimental results (Table S2), which find the number of carbon atoms integrated into the SWCNT to be larger than the number of carbon atoms 
1 ejected from the bulk of the particle by a factor of 2 . This implies that the carbon from the carbide de-

2 composition is forming the tube and the difference suggests that another $\mathrm{C}$ source is contributing to the 3 tube growth.

4 RMD simulations show that a considerable percentage of $\mathrm{C}$ atoms in the catalytic process partic-

5 ipate in surface diffusion, which fluctuates between $15 \%$ and $55 \%$ (Figure S1). Some of these carbon

6 atoms diffusing on the surface may become part of the nanotube as a function of time as illustrated in

7 Figure 4 . The growth rate due to surface diffusion is estimated to be 1.03 carbon atoms $\cdot \mathrm{ns}^{-1}$ from the

8 slope of the straight line that fits the data. These results indicate a constant addition of $\mathrm{C}$ atoms to the

9 nanotube from $\mathrm{C}$ atoms diffusing on the surface, in contrast to the fluctuating behavior originating from

$10 \mathrm{C}$ atoms diffusing out of the particle bulk.

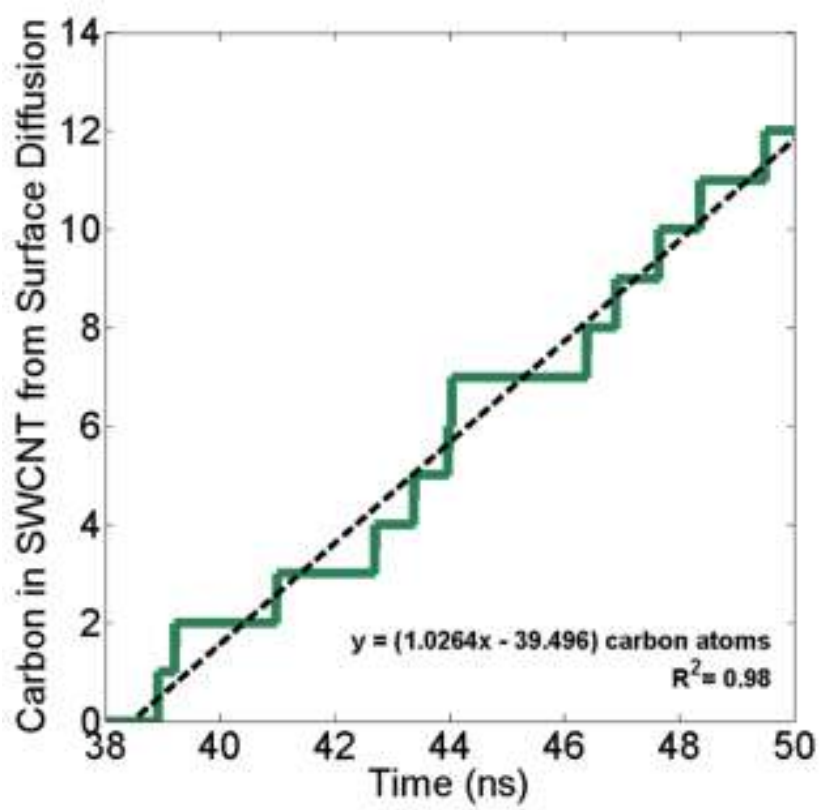

Figure 4. Simulated number of surface diffusing carbon atoms added to SWCNT versus time. The rate of growth due to surface diffusion is estimated to be the slope of a straight line fit to the data relating the number of surface carbon atoms incorporated into the nanotube and time. The fit has a coefficient of determination of 0.98 .

\section{CONCLUSIONS:}


To summarize, both atomic-resolution images and RMD simulations show that two distinct

2 phases; Co and Co-carbide phases co-exist in a single catalyst particle during SWCNT growth. The car-

3 bon-rich phase (carbide) is generally in contact with the support $(\mathrm{MgO})$, and the carbon-poor Co is away

4 from the support and in contact with the SWCNT rim. We propose that the carbon feedstock from the

5 precursor decomposes at the catalyst surface, and part of the resulting carbon diffuses into the particle to

6 form metal carbide. Once the tube forms and partially covers the particle surface, the carbon may not be

7 able to diffuse into the particle efficiently, making the carbide phase unstable. Therefore, a carbon-

8 depleted region that is covered by the tube rim is present during growth. Such a carbon gradient acts as a

9 driving force causing carbon atoms to diffuse over the particle towards the surface. This resolves the

10 mechanism of carbon diffusion in particle sizes below $5 \mathrm{~nm}$. This mechanism is in contrast with the one

11 previously proposed by Baker et al., who suggest that the driving force of carbon diffusion is due to a

12 temperature gradient, despite the fact that it is very difficult to sustain any significant temperature gra-

13 dient in such small particles [42] In the real-time videos, volume ratios of these two phases change as a

14 function of time, consistent with the fluctuating measured SWCNT growth rate. The simulations indi-

15 cate that the rate of tube formation from surface diffusion is constant, while the fluctuating growth ob-

16 served both experimentally and in simulation suggests that bulk processes within the particle are impor-

17 tant. By employing automated image analysis of the large data set (generated by the real-time atomic-

18 resolution videos), we provide first quantitative experimental evidence that the carbon concentration

19 profile developed inside the catalyst is a critical determinant in tube formation, and that the tube growth

20 rate depends on the rates of carbide formation and decomposition. These results are further corroborated

21 by RMD simulations. We also show that this new approach to probe the atomic scale mechanisms for 22 heterogeneous catalysis.

\section{ACKNOWLEDGMENT}


Research (for P. A. Lin and B. Natarajan) was supported by a Cooperative Research Agreement

2 (CRA) between the University of Maryland and the National Institute of Standards and Technology

3 (NIST) (grant 70NANB10H193). The computational work was supported by the US Department of

4 Energy, Basic Energy Sciences, under grant DE-FG02-06ER15836. Computational resources from

5 TAMU Supercomputer Facility, Brazos Cluster at Texas A\&M University, and Texas Advanced Compu-

6 ting Center (TACC) are gratefully acknowledged.

7 Appendix A. Supplementary material. A detailed presentation of RMD results, description of mechan-

8 isms contributing to SWCNT growth, structure determination method and a detailed description of im-

9 age analysis.

10 Appendix B: Movie S1 contains the high resolution video of SWCNT growth from Co catalyst sup11 ported on $\mathrm{MgO}$. 
2 SYNOPSIS TOC: Rational catalyst design requires an atomic scale mechanistic understanding of the 3 chemical pathways involved in the catalytic process. A heterogeneous catalyst typically works by adsorbing reactants onto its surface, where the energies for specific bonds to dissociate and/or combine with other species (to form desired intermediate or final products) are lower. Here, using the catalytic growth of single-walled carbon nanotubes (SWCNTs) as a prototype reaction, we show that the chemical pathway may in-fact involve the entire catalyst particle, and can proceed via the fluctuations in the 8 formation and decomposition of metastable phases in the particle interior. We record in situ and at atom9 ic resolution, the dynamic phase transformations occurring in a Cobalt catalyst nanoparticle during 10 SWCNT growth, using a state-of-the-art environmental transmission electron microscope (ETEM). The 11 fluctuations in catalyst carbon content are quantified by the automated, atomic-scale structural analysis 12 of the time-resolved ETEM images and correlated with the SWCNT growth rate. We find the fluctuations in the carbon concentration in the catalyst nanoparticle and the fluctuations in nanotube growth rates to be of complementary character. These findings are successfully explained by reactive molecular dynamics (RMD) simulations that track the spatial and temporal evolution of the distribution of carbon atoms within and on the surface of the catalyst particle. We anticipate that our approach combining real-time, atomic-resolution image analysis and molecular dynamics simulations will facilitate catalyst design, improving reaction efficiencies and selectivity towards the growth of desired structures.
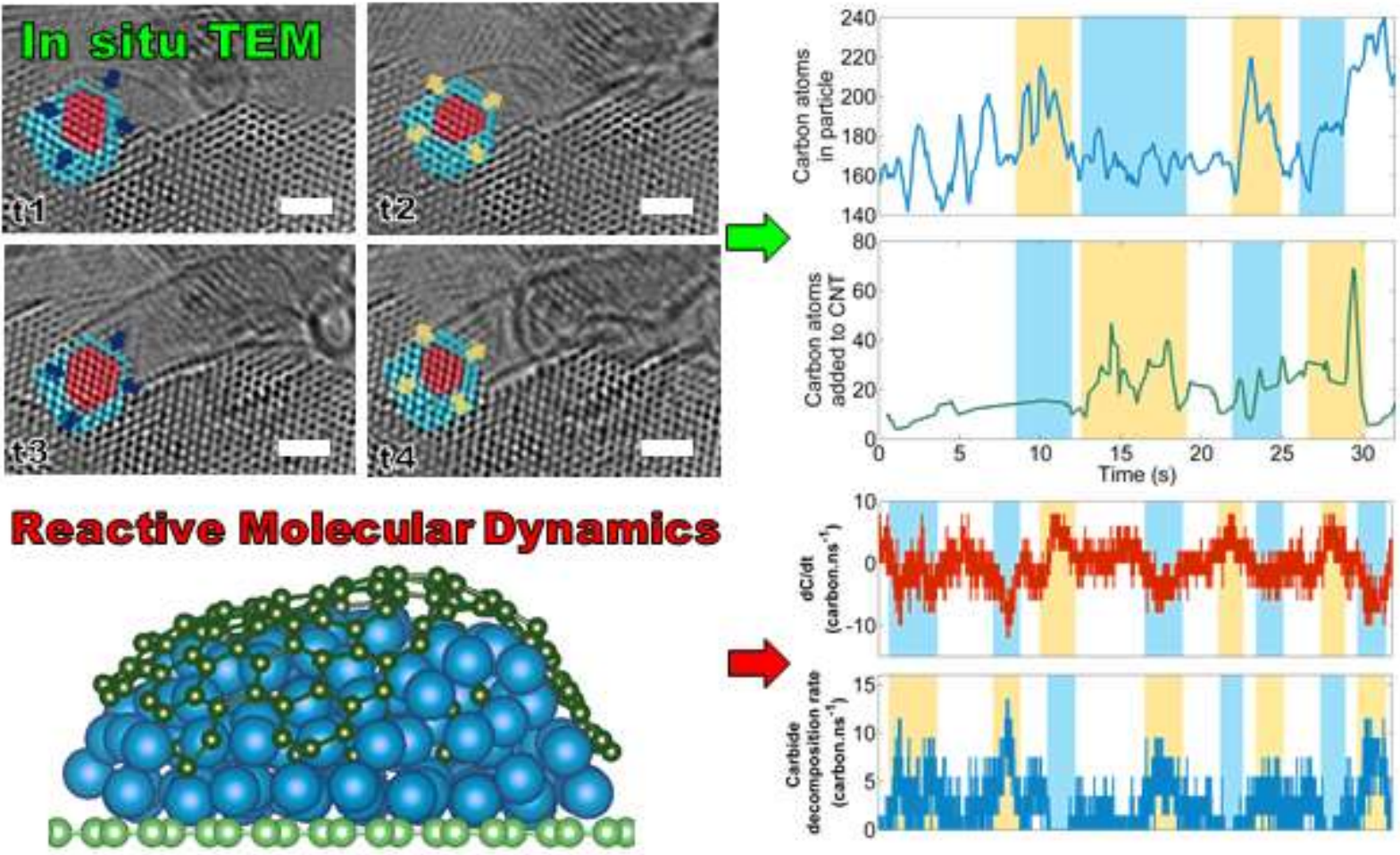


\section{REFERENCES}

[1] J.-C. Charlier, Iijima, Sumio, Growth Mechanisms of Carbon Nanotubes, Springel, Germany, 2001. [2] H. Dai, Rinzler, A.G., Nikolaev, P. Thessm A., Colbert, D.T., Smalley, R.E., Single-wall nanotubes produced by metal-catalyzed disproportionation of carbon monoxide Chemical physics letters, 260 (1996) 471-475.

[3] R.T.K. Baker, Chludzinski, Jr., J.J., Dudash N.S., and Simoens A.J., The formation of filamentous carbon from decomposition of acetylene over vanadium and molybdenum, Carbon, 21 (1983) 463

[4] F. Ding, Bolton, K., Rosen, A., Nucleation and growth of single-walled carbon nanotubes: A molecular dynamics study, Journal of Physical chemistry, B 108 (2004) 17369-17377

[5] R.S. Wagner, Ellis, W.C., Vapor-liquid-solid mechanism of single crystal growth, Applied Physics Letters, 4 (1964) 89-90.

[6] S. Helveg, Lopez-Cartes, C., Sehested, J., Hansen, P.L.,Clausen, B.S., Rostrup-Nielsen, J.R., AbildPedersen, F., and Norskov J., Atomic-scale imaging of carbon nanofibre growth, Nature, 427 (2004) 426

[7] R. Sharma, Zafar, Iqbal In situ observations of carbon nanotube formation using environmental electron microscopy (ETEM), Applied Physics Letters, 84 (2004) 990-992.

[8] S. Hofmann, R. Sharma, C. Ducati, G. Du, C. Mattevi, C. Cepek, M. Cantoro, S. Pisana, A. Parvez, F. Cervantes-Sodi, A.C. Ferrari, R. Dunin-Borkowski, S. Lizzit, L. Petaccia, A. Goldoni, J. Robertson, In situ Observations of Catalyst Dynamics during Surface-Bound Carbon Nanotube Nucleation, Nano Letters, 7 (2007) 602-608.

[9] M. He, H. Jiang, B. Liu, P.V. Fedotov, A.I. Chernov, E.D. Obraztsova, F. Cavalca, J.B. Wagner, T.W. Hansen, I.V. Anoshkin, E.A. Obraztsova, A.V. Belkin, E. Sairanen, A.G. Nasibulin, J. Lehtonen, E.I. Kauppinen, Chiral-selective growth of single-walled carbon nanotubes on lattice-mismatched epitaxial cobalt nanoparticles, Scientific reports, 3 (2013) 1460.

[10] S. Hofmann, R. Blume, C.T. Wirth, M. Cantoro, R. Sharma, C. Ducati, M. Havecker, S. Zafeiratos, P. Schnoerch, A. Oestereich, D. Teschner, M. Albrecht, A. Knop-Gericke, R. Schlogl, J. Robertson, State of Transition Metal Catalysts During Carbon Nanotube Growth, Journal of Physical Chemistry C, 113 (2009) 1648-1656.

[11] S. Mazzucco, Y. Wang, M. Tanase, M. Picher, K. Li, Z.J. Wu, S. Irle, R. Sharma, Direct evidence of active and inactive phases of Fe catalyst nanoparticles for carbon nanotube formation, Journal of Catalysis, 319 (2014) 54-60.

[12] H. Yoshida, S. Takeda, T. Uchiyama, H. Kohno, Y. Homma, Atomic-Scale In-situ Observation of Carbon Nanotube Growth from Solid State Iron Carbide Nanoparticles, Nano Letters, 8 (2008) 20822086.

[13] R. Sharma, Moore, E.S., Rez, P, Treacy M.M.J., Site-specific fabrication of Fe particles for carbon nanotube growth, Nano Letters, 9 (2009) 689-694.

[14] Y. Kohigashi, H. Yoshida, Y. Homma, S. Takeda, Structurally inhomogeneous nanoparticulate catalysts in cobalt-catalyzed carbon nanotube growth, Applied Physics Letters, 105 (2014).

[15] M. Picher, P.A. Lin, J.L. Gomez-Ballesteros, P.B. Balbuena, R. Sharma, Nucleation of Graphene and Its Conversion to Single-Walled Carbon Nanotubes, Nano letters, 14 (2014) 6104-6108.

[16] H. Yoshida, Takeda, Seiji, Uchiyama,Tetsuya, Kohno, Hideo,Homma, Yoshikazu, Atomic-Scale In-situ Observation of Carbon Nanotube Growth from Solid State Iron Carbide Nanoparticles, Nano Letters, 9 (2008) 3810-3815.

[17] J.A. Elliott, Y. Shibuta, H. Amara, C. Bichara, E.C. Neyts, Atomistic modelling of CVD synthesis of carbon nanotubes and graphene, Nanoscale, 5 (2013) 6662-6676.

[18] D.A. Gomez-Gualdron, J.M. Beetge, P.B. Balbuena, Characterization of Metal Nanocatalyst State and Morphology during Simulated Single-Walled Carbon Nanotube Growth, Journal of Physical Chemistry C, 117 (2013) 12061-12070. 
[19] D.A. Gomez-Gualdron, Beetge, J.M., Burgos, J.C., Balbuena, P.B. , Effects of Precursor Type on the CVD Growth of Single-Walled Carbon Nanotubes, Journal of Physical Chemistry C, 117 (2013) 10397-10409.

[20] D.A. Gomez-Gualdron, Balbuena,P.B. , Characterization of Carbon Atomistic Pathways during Single-Walled Carbon Nanotube Growth on Supported Metal Nanoparticles, Carbon, 57 (2013) 298309.

[21] M. Diarra., Zappelli, A., Amara, H., ,Ducastelle, F., Bichara, C., Importance of carbon solubility and wetting properties of nickel nanoparticles for single wall nanotube growth, Physical Review Letters, 109 (2012) 185501.

[22] H. Amara, Roussel, J.-M., Bichara, C., Gaspard, J.-P., Ducastelle, F. , Tight-binding potential for atomistic simulations of carbon interacting with transition metals: Application to the Ni-C system, Physical Review B, 79 (2009) 014109.

[23] U. Khalilov, Bogaerts, A., Neyts, E.C. , Microscopic mechanisms of vertical graphene and carbon nanotube cap nucleation from hydrocarbon growth precursors, Nanoscale, 6 (2014) 9206-9214.

[24] B. Zhang, B.B. Cao, C. Chen, J. Zhang, H.M. Duan, Density-Functional Theory Study on Neutral and Charged MnC2 (M = Fe, Co, Ni, Cu; n=1-5) Clusters, Journal of Cluster Science, 24 (2013) 197207.

[25] A. Martinez-Limia, J. Zhao, P.B. Balbuena, Molecular dynamics study of the initial stages of catalyzed single-wall carbon nanotubes growth: force field development, Journal of Molecular Modeling, 13 (2007) 595-600.

[26] R. Sharma, Crozier, Peter A., Environmental Transmission Electron Microscopy in Nanotechnology, in: N.Y.Z.L. Wang (Ed.) Transmission Electron microscopy for nanotechnology, Springer-Verlag and Tsinghua University Press2005, pp. 531-565.

[27] J.L. Gomez-Ballesteros, Burgos, Juan C., Lin, Pin Ann, Sharmac, Renu, and Balbuena, Perla B. , Nanocatalyst shape and composition during nucleation of single-walled carbon nanotubes, RSC Adv., 5 (2015) 106377-106386.

[28] A.Y. Khodakov, Chu, Wei, Fongarland, Pascal Advances in the Development of Novel Cobalt Fischer-Tropsch Catalysts for Synthesis of Long-Chain Hydrocarbons and Clean Fuels, Chemical Reviews, 107 (2007) 1692-1744.

[29] A. Goyal, D.A. Wiegand, F.J. Owens, Z. Iqbal, Synthesis of carbide-free, high strength iron-carbon nanotube composite by in situ nanotube growth, Chemical physics letters, 442 (2007) 365-371.

32

33 [30] D.W. Brenner, Shenderova, O.A., Harrison, J.A., Stuart, S.J., Ni, B., Sinnott,S.B. , A secondgeneration reactive empirical bond order (REBO) potential energy expression for hydrocarbons, journal of physics: Condensed Matter, 14 (2002) 783.

[31] J. Tersoff, Modeling solid-state chemistry: Interatomic potentials for multicomponent systems, Physical Review B, 39 (1989) 5566-5568.

[32] D.W. Brenner, Empirical potential for hydrocarbons for use in simulating the chemical vapor deposition of diamond films, Physical Review B, 42 (1990) 9458.

[33] A. Sutton, J. Chen, Long-range finnis-sinclair potentials, Philosophical Magazine Letters, 61 (1990) 139-146.

[34] S. Nagakura, Study of metallic carbides by electron diffraction IV. Cobalt carbides, Journal of the Physical Society of Japan, 16 (1961) 1213-1219.

[35] Hussaini, Zahra ,Lin, Pin Ann,, Zhu, Wenhui Natarajan, Bharath, and Sharma, Renu Automated Image Processing Scheme to Measure Atomic-Scale Structural Fluctuations, Microscopy \& Microanalysis 2016, Microscopy Scoiety of America, Columbus OH, 2016, pp. 718-719.

[36] R. Sharma, Rez, Peter, Treacy, M.M.J. and Stuart, Steven J. , In-situ observation of the growth mechanisms of carbon nanotubes under diverse reaction conditions, Journal of Electron Microscopy, 54 (2005) 231-237.

[37] R. Sharma, Rez, P., and Treacy, M.M.J. , Direct observations of the growth of carbon nanotubes using in situ transmission electron microscopy, e-Journal of Surface Science and Nano Technology, 4 51 (2006) 460-463. 
1 [38] K.M. Bal, Neyts, E.C., Merging Metadynamics into Hyperdynamics: Accelerated Molecular

2 Simulations Reaching Time Scales from Microseconds to Seconds, Journal of chemical theory and 3 computation, 11 (2015) 4545-4554.

4 [39] U. Khalilov, Bogaerts, A., Neyts, E.C. , Atomic scale simulation of carbon nanotube nucleation 5 from hydrocarbon precursors, , Nature communications, 6 (2015) 10306.

6 [40] D.A. Gomez-Gualdron, P.B. Balbuena, Characterization of Carbon Atomistic Pathways during

7 Single-Walled Carbon Nanotube Growth on Supported Metal Nanoparticles, Carbon, 57 (2013) 2988309.

9 [41] D.A. Gomez-Gualdron, J.M. Beetge, J.C. Burgos, P.B. Balbuena, Effects of Precursor Type on the 10 CVD Growth of Single-Walled Carbon Nanotubes, Journal of Physical Chemistry C, 117 (2013) 10397 1110409.

12 [42] J. Zhao, Martinez-Limia, A. Balbuena P.B., Understanding catalysed growth of single-wall carbon 13 nanotubes, Nanotechnology, 16 (2005) S575-S581. 



Reactive Molecular Dymamics
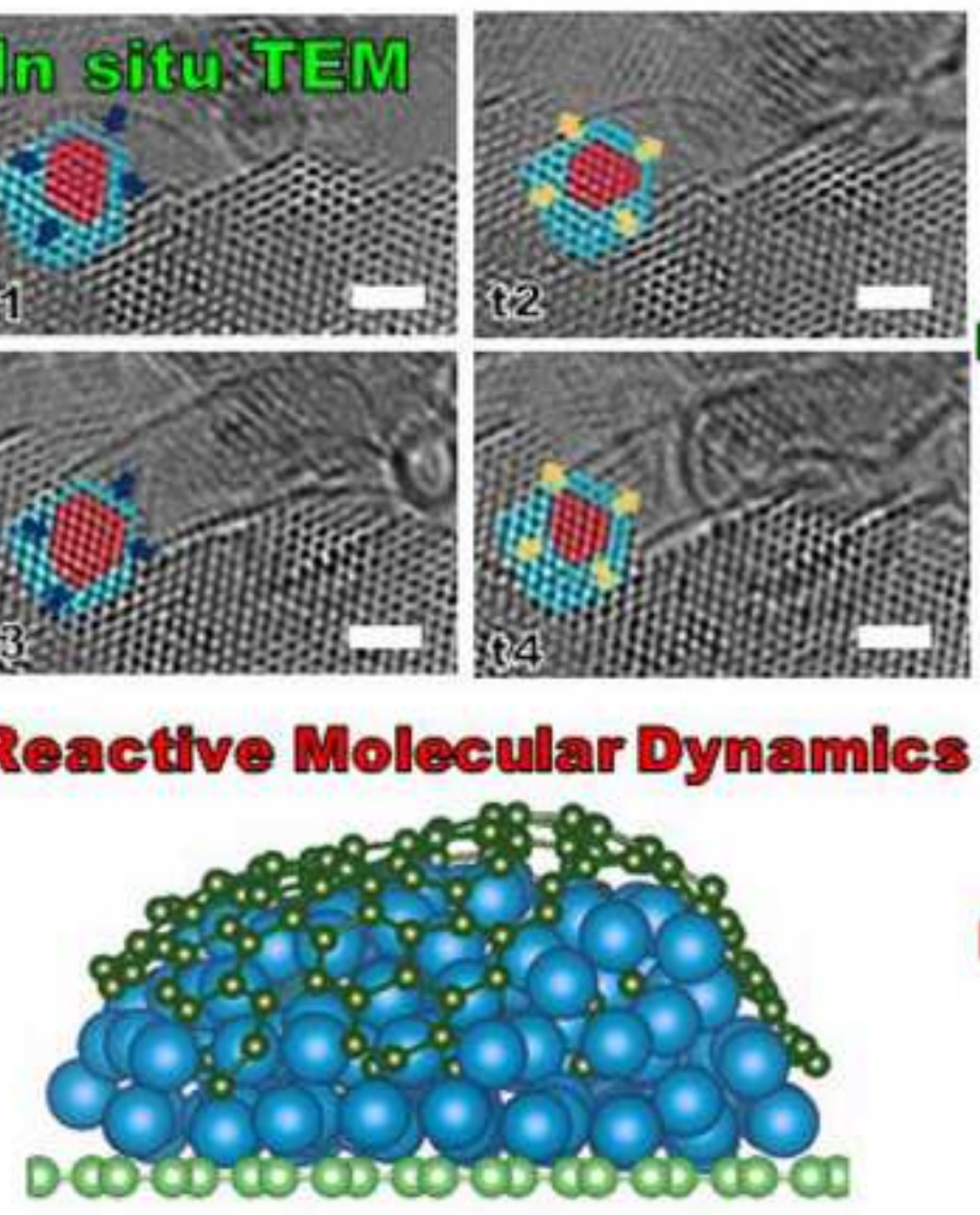
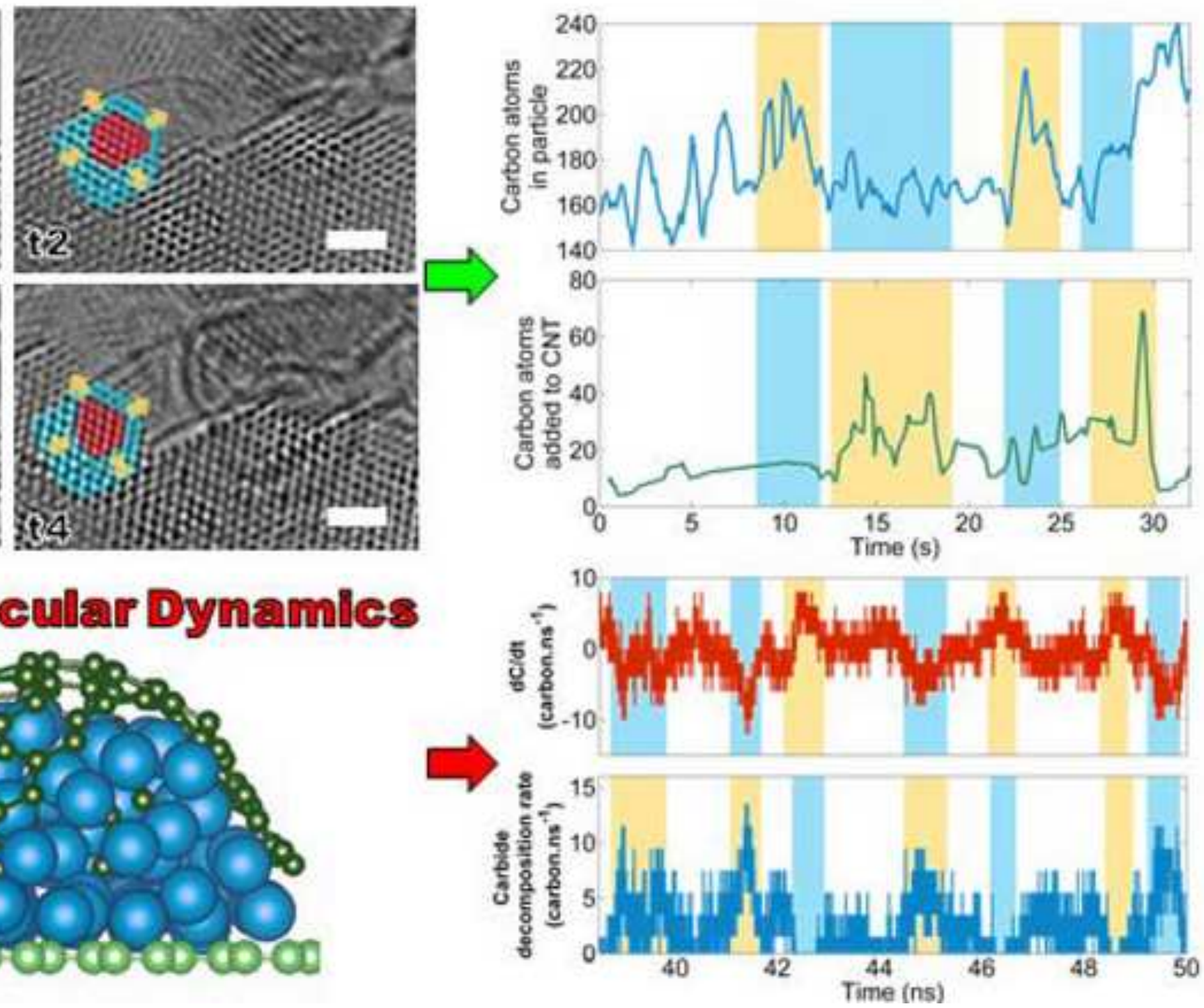